\title{
On The Relative Entropy Method For Hyperbolic-Parabolic Systems
}

\author{
Cleopatra Christoforou and Athanasios Tzavaras
}

\begin{abstract}
The work of [5] on the extension of the relative entropy identity to the class of hyperbolic-parabolic systems whose hyperbolic part is symmetrizable is the context of this article. The general theory is presented and the derivation of the relative entropy identities for both hyperbolic and hyperbolic-parabolic systems is presented. The resulting identities are useful to provide measure valued weak versus strong uniqueness theorems as well as convergence results in the zero-viscosity limit. An application of this theory is given for the example of the system of thermoviscoelasticity.
\end{abstract}

\section{The Relative Entropy Method}

This manuscript serves as a review article of the relative entropy method as it has been recently extended in the work of Christoforou and Tzavaras in [?] for general systems of hyperbolic-parabolic conservation laws

$$
\partial_{t} A(u)+\partial_{\alpha} F_{\alpha}(u)=\varepsilon \partial_{\alpha}\left(B_{\alpha \beta}(u) \partial_{\beta} u\right) .
$$

Here $u(t, x)$ takes values in $\mathbb{R}^{n}, t \in \mathbb{R}^{+}, x \in \mathbb{R}^{d}$ with $n, d$ being integers representing the number of the conserved quantities and the space dimension. The functions $A, F_{\alpha}: \mathbb{R}^{n} \rightarrow \mathbb{R}^{n}, B_{\alpha \beta}: \mathbb{R}^{n} \rightarrow \mathbb{R}^{n \times n}$ are smooth, $\alpha, \beta=1, \ldots, d$, and the summa-

Cleopatra Christoforou

Department of Mathematics and Statistics, University of Cyprus, Nicosia 1678, Cyprus, e-mail: christoforou.cleopatra@ucy.ac.cy

Athanasios Tzavaras

Computer, Electrical, Mathematical Sciences \& Engineering Division, King Abdullah University of Science and Technology (KAUST), Thuwal, Saudi Arabia. e-mail: athanasios.tzavaras@kaust.edu.sa and Institute of Applied and Computational Mathematics, FORTH, Heraklion, Greece. 
tion convention over repeated indices is employed throughout the article. Also it is assumed that the associated hyperbolic problem

$$
\partial_{t} A(u)+\partial_{\alpha} F_{\alpha}(u)=0
$$

is symmetrizable in the sense of Friedrichs and Lax [17].

The idea of relative entropy, introduced by Dafermos [8,9] and DiPerna [12], is quite powerful in comparing solutions of conservation laws (e.g. [12, 3, 11, 24]), or balance laws (e.g. [27, 23]), and has recently being applied to problems that are classified under the domain of hyperbolic-parabolic systems (e.g [14, 21, 22, 4]). The aim is to extend the class of computations that go under the general term relative entropy to the broader class of systems (2) and (1) and to systematize the derivation of relative entropy identities in a unifying framework. Moreover, the connection of the relative entropy theory with its natural framework, the $L^{2}$ theory of hyperbolicparabolic systems of Kawashima [19] and the developments on Green functions by Liu-Zeng [28] is shown and in particular with the framework of thermodynamics. It should be emphasised that from the very early developments of the method $[8,9,18]$ the theory appears in the context of thermodynamics. Therefore in the end of this article, this connection is revisited in the context of the general constitutive theory of thermoviscoelasticity, whose thermodynamical structure is specified in $[6,7,26]$.

Hypotheses on the constitutive functions and the viscosity matrices that characterise the class of systems (1) and (2) for which the relative entropy method is extended are the following:

$\left(\mathrm{H}_{1}\right) \quad A: \mathbb{R}^{n} \rightarrow \mathbb{R}^{n}$ is a $C^{2}$ globally invertible map,

$\left(\mathrm{H}_{2}\right)$ existence of an entropy-entropy flux pair $(\eta, q)$, that is $\exists G: \mathbb{R}^{n} \rightarrow \mathbb{R}^{n}, G=G(u)$ smooth such that

$$
\begin{aligned}
\nabla \eta & =G \cdot \nabla A \\
\nabla q_{\alpha} & =G \cdot \nabla F_{\alpha}, \quad \alpha=1, \ldots, d,
\end{aligned}
$$

$\left(\mathrm{H}_{3}\right)$ the matrix $\nabla^{2} \eta(u)-G(u) \cdot \nabla^{2} A(u)$ is symmetric and positive definite,

$\left(\mathrm{H}_{4}\right)$ the matrix $\sum_{\alpha, \beta} \nabla G(u)^{T} B_{\alpha \beta}(u)$ satisfies Kawashima condition; the matrix $\sum_{\alpha, \beta} \nabla G(u)^{T} B_{\alpha \beta}(u) \omega_{\alpha} \omega_{\beta}$ is real symmetric, positive semi-definite for all $\omega \in$ $S^{d-1}$,

$$
\sum_{\alpha, \beta=1}^{d} \xi_{\alpha}^{T} \nabla G(u)^{T} B_{\alpha \beta}(u) \xi_{\beta} \geq \gamma\|\xi\|, \quad \forall \xi_{\alpha}, \xi_{\beta} \in \mathbb{R}^{n} .
$$

These hypotheses are set and guided by the goal of rendering the relative entropy identity useful and applying it to some standard problems. Let us add that Hypotheses $\left(\mathrm{H}_{1}\right)-\left(\mathrm{H}_{3}\right)$ are equivalent to the usual symmetrizability hypothesis in the sense of Friedrichs and Lax and therefore, they render system (2) hyperbolic. The additional hypotheses $\left(\mathrm{H}_{4}\right)$ for the hyperbolic-parabolic systems (1) guarantees that the entropy dissipates along the evolution. In [?], we prolong the relative entropy method to this broader class of systems and derive the relative entropy identities for systems (1) and (2). The derivation of these identities (19) and (20) is presented in Section 2. 
The structure of this manuscript is as follows: In Section 2 we state the main hypotheses that make the relative entropy into a workable quantity, and establish their connections to the theory of symmetrizable systems [17] and to the $L^{2}$ theory of hyperbolic-parabolic systems [19]. We then derive the relative entropy identities in Section 3 for both hyperbolic systems (2) and hyperbolic-parabolic systems (1) and see that the usual dissipative structure for hyperbolic-parabolic systems suffices to control the various error terms that appear. We conclude in Section 4 by undertaking this issue in the context of a specific application. We take up the system of thermoviscoelasticity in several space dimensions under its constitutive theory. We derive the relative entropy identity that is pertinent to this theory and describe how the general theory for hyperbolic-parabolic systems takes particular shape when applied to the constitutive theory of thermoviscoelasticity. Related formulas in more special situations have been computed in $[14,15]$ for gases with Stokes viscosity and Fourier heat conduction and in [18] for the constitutive theory of thermoelasticity. It should be noted that for this example, the convexity of the entropy in the conserved variables translates into the usual thermodynamic stability conditions $\psi_{F F}(F, \theta)>0$ and $\eta_{\theta}(F, \theta)>0$ familiar from the work of Gibbs for a theory with thermal and elastic effects.

The identities derived in the general theory in Section 2 or the example in thermoviscoelasticity in Section 3 are quite powerful in establishing weak versus strong uniqueness theorems, stability of bounded smooth solutions of hyperbolic-parabolic systems as well as convergence results in the zero viscosity limit to a smooth solution of inviscid system (2). Several propositions in each setting are proven in [5] using the relative entropy identities. However these are not presented in this review article. We refer the reader to [5] to realise how these identities are exploited in these theorems. Let us only add that an interesting feature of the analysis is how concentration measures are defined for a symmetrizable hyperbolic system (2) and the associated form of the averaged relative entropy identity. Last, systems of balance laws are also studied in [5] and the role of the source terms in the derivation of the relative entropy identity is investigated in the proof of the weak-strong uniqueness result.

\section{The General Framework}

Consider first the constituent system of conservation laws (2) and assume that $A$ : $\mathbb{R}^{n} \rightarrow \mathbb{R}^{n}$ is a $C^{2}$ map which is one-to-one and satisfies

$$
\nabla A(u) \text { is nonsingular } \forall u \in \mathbb{R}^{n} .
$$

By the inverse function theorem the map $v=A(u)$ is locally invertible with the inverse map $u=A^{-1}(v)$ a $C^{2}$ map.

Next, a structural hypothesis is added that is the system (2) is endowed with an additional conservation law 


$$
\partial_{t} \eta(u)+\partial_{\alpha} q_{\alpha}(u)=0 .
$$

Indeed this is expressed as follows: The functions $\eta-q, q=\left(q_{\alpha}\right), \alpha=1, \ldots, d$, are called an entropy pair ( $\eta$ is called entropy and $q=\left(q_{\alpha}\right)$, the associated entropy-flux) if there exists a smooth function $G: \mathbb{R}^{n} \rightarrow \mathbb{R}^{n}, G=G(u)$, such that simultaneously

$$
\begin{aligned}
\nabla \eta & =G \cdot \nabla A \\
\nabla q_{\alpha} & =G \cdot \nabla F_{\alpha}, \quad \alpha=1, \ldots, d .
\end{aligned}
$$

If $\left(\mathrm{H}_{2}\right)$ is satisfied then smooth solutions of (2) satisfy the additional identity (3). One checks that $\left(\mathrm{H}_{2}\right)$ is equivalent to requiring that $G$ satisfies the simultaneous equations

$$
\begin{aligned}
\nabla G^{T} \nabla A & =\nabla A^{T} \nabla G \\
\nabla G^{T} \nabla F_{\alpha} & =\nabla F_{\alpha}{ }^{T} \nabla G, \quad \alpha=1, \ldots, d .
\end{aligned}
$$

That is, if there exists a multiplier $G(u)$ satisfying $\left(\mathrm{H}_{2}\right)$ (equivalently (4), (5)) then (2) is endowed with the additional conservation law (3) and it is well known that systems from mechanics naturally inherit the entropy pair structure from the second law of thermodynamics.

Given two solutions $u, \bar{u}$ of (2), the relative entropy is defined via

$$
\eta(u \mid \bar{u})=\eta(u)-\eta(\bar{u})-G(\bar{u}) \cdot(A(u)-A(\bar{u}))
$$

while the relative flux(es) by

$$
q_{\alpha}(u \mid \bar{u})=q_{\alpha}(u)-q_{\alpha}(\bar{u})-G(\bar{u}) \cdot\left(F_{\alpha}(u)-F_{\alpha}(\bar{u})\right) .
$$

Formula (6) will be used to estimate the distance between two solutions $u$ and $\bar{u}$. To make it amenable to analysis, we note that $\nabla^{2} \eta(u)-G(u) \cdot \nabla^{2} A(u)$ is symmetric and require that it is positive definite, that is

$$
\xi \cdot\left(\nabla^{2} \eta(u)-G(u) \cdot \nabla^{2} A(u)\right) \xi>0 \text { for } \xi \in \mathbb{R}^{n} \backslash\{0\} .
$$

Under $\left(\mathrm{H}_{3}\right)$, expression (6) is useful for comparing the distance between two solutions $u(t, x)$ and $\bar{u}(t, x)$. The definition of relative entropy and flux(es) given by (6)-(7) extends to the case of system (2) a well known definition pursued in [8, 12] for the case $A(u)=u$ with the same objective of calculating the distance between two solutions. Also in [19], one can find a special case of the quantity (6) for comparing a general solution $u(t, x)$ to a constant state $\bar{u}$, in connection to asymptotic behavior problems.

Regarding the hyperbolic-parabolic system (1), in addition to hypotheses $\left(\mathrm{H}_{1}\right)$, $\left(\mathrm{H}_{2}\right),\left(\mathrm{H}_{3}\right)$ on the hyperbolic part, we assume that the viscosity matrices that induce a dissipative structure. Using the multiplier $G(u)$ in $\left(\mathrm{H}_{2}\right)$, we deduce that smooth solutions of (1) satisfy the identity 
On The Relative Entropy Method For Hyperbolic-Parabolic Systems

$$
\partial_{t} \eta(u)+\partial_{\alpha} q_{\alpha}(u)=\varepsilon \partial_{\alpha}\left(G(u) \cdot B_{\alpha \beta}(u) \partial_{\beta} u\right)-\varepsilon \nabla G(u) \partial_{\alpha} u \cdot B_{\alpha \beta}(u) \partial_{\beta} u .
$$

We will require that the entropy dissipates along the evolution, namely that the matrix $\sum_{\alpha, \beta} \nabla G(u)^{T} B_{\alpha \beta}(u) \omega_{\alpha} \omega_{\beta}$ is real (symmetric) positive semi-definite for all $\omega \in S^{d-1}$,

$$
\begin{aligned}
& \nabla G(u)^{T} B_{\alpha \beta}(u)=B_{\alpha \beta}(u)^{T} \nabla G(u) \\
& \sum_{\alpha, \beta=1}^{d} \xi_{\alpha} \cdot \nabla G(u)^{T} B_{\alpha \beta}(u) \xi_{\beta} \geq 0 \quad \forall \xi_{\alpha}, \xi_{\beta} \in \mathbb{R}^{n},
\end{aligned}
$$

Hypothesis $\left(\mathrm{H}_{4}\right)$ is natural in the context of applications to mechanics as it is connected to entropy dissipation and the Clausius-Duhem inequality. When exploiting the identities, we often impose a strengthened version of $\left(\mathrm{H}_{4}\right)$ :

$$
\begin{gathered}
\nabla G(u)^{T} B_{\alpha \beta}(u)=B_{\alpha \beta}(u)^{T} \nabla G(u) \\
\sum_{\alpha, \beta=1}^{d} \xi_{\alpha} \cdot \nabla G(u)^{T} B_{\alpha \beta}(u) \xi_{\beta}>0 .
\end{gathered}
$$

$\forall \xi_{\alpha}, \xi_{\beta} \in \mathbb{R}^{n} \backslash\{0\}$. The minimum $v(u)$ and maximum $N(u)$ eigenvalues of the associated quadratic form, for $u \in \mathbb{R}^{n}$, may be used to express $\left(\mathrm{H}_{4 s}\right)$ in an equivalent (more quantitative) format:

$$
0<v(u)|\xi|^{2} \leq \sum_{\alpha, \beta=1}^{d} \xi_{\alpha} \cdot \nabla G(u)^{T} B_{\alpha \beta}(u) \xi_{\beta} \leq N(u)|\xi|^{2}, \quad\left(\mathrm{H}_{4 p}\right)
$$

$\forall \xi \in \mathbb{R}^{n}$.

Remark 1. We can compare the Hypotheses $\left(\mathrm{H}_{2}\right)$ and $\left(\mathrm{H}_{3}\right)$ with the familiar notion of symmetrizable first-order systems of Friedrichs and Lax [17] using the transformation $v=A(u)$ since this is invertible by Hypothesis $\left(\mathrm{H}_{1}\right)$. Then systems (2) and (3) can be expressed in terms of the conserved variables $v$,

$$
\begin{aligned}
\partial_{t} v+\partial_{\alpha}\left(F_{\alpha} \circ A^{-1}\right)(v) & =0 \\
\partial_{t}\left(\eta \circ A^{-1}\right)(v)+\partial_{\alpha}\left(q_{\alpha} \circ A^{-1}\right)(v) & =0 .
\end{aligned}
$$

Setting

$$
f_{\alpha}(v)=F_{\alpha} \circ A^{-1}(v), \quad H(v)=\eta \circ A^{-1}(v), \quad Q_{\alpha}(v)=q_{\alpha} \circ A^{-1}(v) .
$$

we obtain the formulas

$$
\eta(u)=H(A(u)), \quad q_{\alpha}(u)=Q_{\alpha}(A(u)) .
$$

One can easily check that $\left(\mathrm{H}_{2}\right)$ is equivalent to

$$
\nabla_{v} Q_{\alpha}(v)=\nabla_{v} H(v) \cdot \nabla_{v} f_{\alpha}(v),
$$


and

$$
G(u)=\left(\nabla_{v} H\right)(A(u))
$$

Hence $\eta-q$ is an entropy pair for (2) satisfying $\left(\mathrm{H}_{2}\right)$ if and only if the pair $H-Q$ is an entropy pair for $(9)$ satisfying $\left(\mathrm{h}_{2}\right)$. Also Hypothesis $\left(\mathrm{H}_{3}\right)$ translates to the requirement that the entropy $H(v)$ is convex,

$$
\zeta \cdot \nabla_{v}^{2} H(v) \zeta>0 \quad \text { for } \zeta \in \mathbb{R}^{n}, \zeta \neq 0 .
$$

In summary, $\left(\mathrm{H}_{1}\right),\left(\mathrm{H}_{2}\right)$ and $\left(\mathrm{H}_{3}\right)$ are equivalent to the usual symmetrizability hypothesis of [17]. To study the meaning of Hypothesis $\left(\mathrm{H}_{4}\right)$ first rewrite (1) and multiply by $\nabla G(u)^{T}$ to get

$$
\begin{array}{r}
\nabla G(u)^{T} \nabla A(u) \partial_{t} u+\nabla G(u)^{T} \nabla F_{\alpha}(u) \partial_{\alpha} u=\varepsilon \nabla G(u)^{T} B_{\alpha \beta}(u) \partial_{\alpha} \partial_{\beta} u \\
+\varepsilon \nabla G(u)^{T} \nabla B_{\alpha \beta}(u):\left(\partial_{\alpha} u, \partial_{\beta} u\right) .
\end{array}
$$

Hypothesis $\left(\mathrm{H}_{4}\right)$ implies that the diffusion coefficient matrix $\sum_{\alpha, \beta} \tilde{B}_{\alpha \beta}(u) \omega_{\alpha} \omega_{\beta}=$ $\sum \nabla G(u)^{T} B_{\alpha \beta}(u) \omega_{\alpha} \omega_{\beta}$ is symmetric and positive semi-definite satisfying

$$
\tilde{B}_{\alpha \beta}(u)=\tilde{B}_{\alpha \beta}(u)^{T}, \quad \sum_{\alpha, \beta} \xi_{\alpha} \cdot \tilde{B}_{\alpha \beta}(u) \xi_{\beta} \geq 0,
$$

$\forall \xi_{\alpha}, \xi_{\beta} \in \mathbb{R}^{n}$. This hypothesis is connected to the local well-posedness of hyperbolicparabolic systems (1), cf. [19, Ch II] and [10].

Remark 2. The analysis by Christoforou and Tzavaras [5] is not a direct application of the change of variable $v=A(u)$ to the existing theory for $A(u)=u$ that one might at a first glance believe. Indeed the theory for hyperbolic-parabolic systems cannot be studied only based on the change of variables due to the viscous part of the system as one can see from the computation of the previous remark. This raised the need to extend the relative entropy method to a more general framework for systems (1) and (2).

\section{The relative entropy identity}

In this section we extend a well known calculation developed in $[8,12]$ for the case $A(u)=u$ to the hyperbolic system (2), subject to hypotheses $\left(\mathrm{H}_{1}\right),\left(\mathrm{H}_{2}\right)$ and $\left(\mathrm{H}_{3}\right)$.

Let $u$ be an entropy weak solution of (2), that is $u$ is a weak solution of (2) that satisfies in the sense of distributions the inequality

$$
\partial_{t} \eta(u)+\partial_{\alpha} q_{\alpha}(u) \leq 0 .
$$

Let $\bar{u}$ be a strong (conservative) solution of (2) that is satisfying the entropy identity 


$$
\partial_{t} \eta(\bar{u})+\partial_{\alpha} q_{\alpha}(\bar{u})=0
$$

We proceed to compute the relative entropy identity for the quantities relative entropy (6) and relative flux (7). Observe first that $u, \bar{u}$ satisfy the chain of identities

$$
\begin{aligned}
\partial_{t}(G(\bar{u}) \cdot(A(u)-A(\bar{u})))+ & \partial_{\alpha}\left(G(\bar{u}) \cdot\left(F_{\alpha}(u)-F_{\alpha}(\bar{u})\right)\right) \\
= & \nabla G(\bar{u}) \partial_{t} \bar{u} \cdot(A(u)-A(\bar{u}))+\nabla G(\bar{u}) \bar{u}_{x_{\alpha}} \cdot\left(F_{\alpha}(u)-F_{\alpha}(\bar{u})\right) \\
= & -\bar{u}_{x_{\alpha}} \cdot \nabla F_{\alpha}(\bar{u})^{T} \nabla A(\bar{u})^{-T} \nabla G(\bar{u})^{T}(A(u)-A(\bar{u})) \\
& +\nabla G(\bar{u}) \bar{u}_{x_{\alpha}} \cdot\left(F_{\alpha}(u)-F_{\alpha}(\bar{u})\right) \\
= & -\nabla G(\bar{u}) \bar{u}_{x_{\alpha}} \cdot \nabla F_{\alpha}(\bar{u}) \nabla A(\bar{u})^{-1}(A(u)-A(\bar{u})) \\
& +\nabla G(\bar{u}) \bar{u}_{x_{\alpha}} \cdot\left(F_{\alpha}(u)-F_{\alpha}(\bar{u})\right) \\
= & : \nabla G(\bar{u}) \bar{u}_{x_{\alpha}} \cdot F_{\alpha}(u \mid \bar{u})
\end{aligned}
$$

where

$$
F_{\alpha}(u \mid \bar{u}):=F_{\alpha}(u)-F_{\alpha}(\bar{u})-\nabla F_{\alpha}(\bar{u}) \nabla A(\bar{u})^{-1}(A(u)-A(\bar{u})) .
$$

Combining (15), (16) and (17), we obtain

$$
\partial_{t} \eta(u \mid \bar{u})+\partial_{\alpha} q_{\alpha}(u \mid \bar{u}) \leq-\partial_{\alpha} G(\bar{u}) \cdot F_{\alpha}(u \mid \bar{u}) .
$$

The above calculation is formal, it can however be made rigorous following ideas that are well developed (see e.g. [10, Ch V]) and provides a way of comparing a weak entropic to a strong solution of (2). There exist variants of this calculation that compare entropic measure valued solutions to strong solutions of hyperbolic conservation laws (see $[3,11,5])$.

An analogous but more complicated analysis leads us to the relative entropy identity

$$
\begin{aligned}
\partial_{t} \eta(u \mid \bar{u}) & +\partial_{\alpha} q_{\alpha}(u \mid \bar{u})+\varepsilon \nabla G(u) \partial_{\alpha}(u-\bar{u}) \cdot B_{\alpha \beta}(u) \partial_{\beta}(u-\bar{u}) \\
& =-\nabla G(\bar{u})\left(\partial_{\alpha} \bar{u}\right) \cdot F_{\alpha}(u \mid \bar{u})+\varepsilon \partial_{x_{\alpha}}\left(J_{\alpha}+j_{\alpha}\right)+\varepsilon \sum_{i=1}^{6} Q_{i}
\end{aligned}
$$

among two solutions $u$ and $\bar{u}$ of (1) under the framework $\left(\mathrm{H}_{1}\right)-\left(\mathrm{H}_{4}\right)$. Here the viscous fluxes $J_{\alpha}$ and $j_{\alpha}$ are

$$
\begin{gathered}
J_{\alpha}=(G(u)-G(\bar{u})) \cdot\left(B_{\alpha \beta}(u) u_{x_{\beta}}-B_{\alpha \beta}(\bar{u}) \bar{u}_{x_{\beta}}\right)+B_{\alpha \beta}(\bar{u}) \bar{u}_{x_{\beta}} \cdot G(u \mid \bar{u}), \\
j_{\alpha}:=B_{\alpha \beta}(\bar{u}) \bar{u}_{x_{\beta}} \cdot \nabla G(\bar{u}) \phi(u \mid \bar{u}),
\end{gathered}
$$

with

$$
G(u \mid \bar{u}):=G(u)-G(\bar{u})-\nabla G(\bar{u}) \nabla A(\bar{u})^{-1}(A(u)-A(\bar{u})) .
$$

while $Q_{i}$ represent quadratic "error" terms. The details of this derivation can be found in Section 2.4 of [5]. 


\section{Application in Thermoviscoelasticity}

Here we present the relative entropy calculation for the system of thermoviscoelasticity in several space dimensions. In [5] this calculation is also performed for the system of thermoviscoelasticity when restricted to one-space dimension (when restricted to the particular case of Stokes viscosity and Fourier heat conduction). The requirements imposed from thermodynamics on the constitutive theory of thermoviscoelasticity were developed in $[6,7]$ and a summary can be found in $[10$, Sec 3.2]. The constitutive theory takes the form

$$
\begin{aligned}
\psi & =\psi(F, \theta), \\
\Sigma & =\frac{\partial \psi}{\partial F}(F, \theta), \\
\eta & =-\frac{\partial \psi}{\partial \theta}(F, \theta), \\
e & =\psi+\theta \eta .
\end{aligned}
$$

The total stress is decomposed into an elastic part $\Sigma$ and a viscoelastic part $Z=$ $Z(F, \theta, G, \dot{\bar{F}})$ where $\Sigma$ and $Z$ are both symmetric tensor valued functions, $Z(F, \theta, 0,0)=$ 0 so that

$$
\begin{aligned}
S & =\Sigma(F, \theta)+Z(F, \theta, G, \dot{\bar{F}}) \\
& =\frac{\partial \psi}{\partial F}(F, \theta)+Z(F, \theta, G, \dot{\bar{F}}), \\
Q & =Q(F, \theta, G) .
\end{aligned}
$$

Moreover, the heat flux $Q$ and the viscoelastic contribution to the stress $Z$ have to satisfy

$$
\frac{1}{\theta} G \cdot Q(F, \theta, G)+\dot{\bar{F}}: Z(F, \theta, G, \dot{\bar{F}}) \geq 0 \quad \forall(F, \theta, G, \dot{\bar{F}}),
$$

which along with (24) guarantee consistency for smooth processes with the ClausiusDuhem inequality $[6,7]$.

For simplicity we assume that $Z=Z(F, \theta, \dot{\bar{F}})$ i.e. $Z$ is taken independent of $G=$ $\nabla \theta$. Hence condition $(\mathrm{H})$ implies $Q(F, \theta, 0)=0, Z(F, \theta, 0)=0$, and accordingly $(\mathrm{H})$ decomposes into two distinct inequalities

$$
\frac{1}{\theta} G \cdot Q(F, \theta, G) \geq 0 \quad \text { and } \quad \dot{\bar{F}}: Z(F, \theta, \dot{\bar{F}}) \geq 0 .
$$

The system of thermoviscoelasticity then takes the form

$$
\begin{aligned}
F_{t} & =\nabla v \\
v_{t} & =\operatorname{div}(\Sigma+Z)+f \\
\partial_{t}\left(\frac{1}{2}|v|^{2}+e\right) & =\operatorname{div}(v \cdot \Sigma+v \cdot Z)+\operatorname{div} Q+v \cdot f+r .
\end{aligned}
$$


Recall that $x$ stands for the Lagrangean variable, div is the usual divergence operator (in spatial coordinates), while $\partial_{t}$ is here the material derivative, hence $F_{t}=\dot{\bar{F}}$. One checks that smooth solutions of (25) satisfy the energy dissipation identity

$$
\partial_{t} e=\nabla v:(\Sigma+Z)+\operatorname{div} Q+r
$$

and the entropy production identity

$$
\partial_{t} \eta-\operatorname{div} \frac{Q}{\theta}=\frac{1}{\theta^{2}} \nabla \theta \cdot Q+\frac{1}{\theta} \nabla v: Z+\frac{r}{\theta} .
$$

To relate now to the general theory of the previous sections, we set

$$
U=\left(\begin{array}{c}
F \\
v \\
\theta
\end{array}\right) \in \mathbb{R}^{d^{2}+d+1}, \quad A(U)=\left(\begin{array}{c}
F \\
v \\
\frac{1}{2} v^{2}+e(F, \theta)
\end{array}\right)
$$

and impose $e_{\theta}(F, \theta)>0$ so that $A(U)$ is invertible. Also we set

$$
\hat{\eta}(U):=-\eta(F, \theta)
$$

where $\hat{\eta}(U)$ is the mathematical entropy and $\eta(u, \theta)$ the thermodynamic one. Here the relative entropy identity takes the form

$$
\begin{aligned}
\hat{\eta}(U \mid \bar{U}) & =-\eta(F, \theta)+\eta(\bar{F}, \bar{\theta})-\frac{1}{\bar{\theta}}(\bar{\Sigma}, \bar{v},-1) \cdot\left(F-\bar{F}, v-\bar{v}, e(F, \theta)+\frac{1}{2}|v|^{2}-e(\bar{F}, \bar{\theta})-\frac{1}{2}|\bar{v}|^{2}\right) \\
& \stackrel{(24)}{=} \frac{1}{\bar{\theta}}\left[\psi(F, \theta \mid \bar{F}, \bar{\theta})+\frac{1}{2}|v-\bar{v}|^{2}+(\eta(F, \theta)-\eta(\bar{F}, \bar{\theta}))(\theta-\bar{\theta})\right] .
\end{aligned}
$$

We use the notation

$$
\begin{aligned}
\psi(F, \theta \mid \bar{F}, \bar{\theta}) & =\psi(F, \theta)-\psi(\bar{F}, \bar{\theta})-\frac{\partial \psi}{\partial F}(\bar{F}, \bar{\theta}):(F-\bar{F})-\frac{\partial \psi}{\partial \theta}(\bar{F}, \bar{\theta})(\theta-\bar{\theta}) \\
& =\psi-\bar{\psi}-\bar{\Sigma}:(F-\bar{F})+\bar{\eta}(\theta-\bar{\theta})
\end{aligned}
$$

and set $\bar{\psi}=\psi(\bar{F}, \bar{\theta}), \bar{\eta}=\eta(\bar{F}, \bar{\theta})$ and so on. We see that

$$
\nabla^{2} \hat{\eta}(U)-G(U) \cdot \nabla^{2} A(U) \stackrel{(24)}{=}\left(\begin{array}{ccc}
\frac{1}{\theta} \psi_{F F} & 0 & 0 \\
0 & \frac{1}{\theta} & 0 \\
0 & 0 & \frac{1}{\theta} \eta_{\theta}
\end{array}\right)
$$

and the positivity for the matrix $\nabla^{2} \hat{\eta}(U)-G(U) \cdot \nabla^{2} A(U)$ is equivalent to the usual Gibbs thermodynamic stability conditions $\psi_{F F}>0$ and $\eta_{\theta}>0$.

A careful analysis in a similar fashion as in Section 3 leads to the relative entropy identity or the system of thermoviscoelasticity (25) 


$$
\begin{aligned}
& \partial_{t}\left(\psi(F, \theta \mid \bar{F}, \bar{\theta})+(\eta-\bar{\eta})(\theta-\bar{\theta})+\frac{1}{2}|v-\bar{v}|^{2}\right) \\
& -\operatorname{div}\left((v-\bar{v}) \cdot(\Sigma+Z-\bar{\Sigma}-\bar{Z})+(\theta-\bar{\theta})\left(\frac{Q}{\theta}-\frac{\bar{Q}}{\bar{\theta}}\right)\right) \\
& =-\bar{\theta}_{t} \eta(F, \theta \mid \bar{F}, \bar{\theta})+\bar{F}_{t}: \Sigma(F, \theta \mid \bar{F}, \bar{\theta}) \\
& \quad-\theta \bar{\theta}\left(\frac{\nabla v}{\theta}-\frac{\nabla \bar{v}}{\bar{\theta}}\right):\left(\frac{Z}{\theta}-\frac{\bar{Z}}{\bar{\theta}}\right)-\left(\bar{\theta} \frac{Q}{\theta}-\theta \frac{\bar{Q}}{\bar{\theta}}\right) \cdot\left(\frac{\nabla \theta}{\theta}-\frac{\nabla \bar{\theta}}{\bar{\theta}}\right) \\
& \quad+(v-\bar{v}) \cdot(f-\bar{f})+(\theta-\bar{\theta})\left(\frac{r}{\theta}-\frac{\bar{r}}{\bar{\theta}}\right) .
\end{aligned}
$$

Here we have set

$$
\begin{aligned}
\eta(F, \theta \mid \bar{F}, \bar{\theta}) & :=\eta(F, \theta)-\eta(\bar{F}, \bar{\theta})+\frac{\partial \Sigma_{i \alpha}}{\partial \theta}(\bar{F}, \bar{\theta})\left(F_{i \alpha}-\bar{F}_{i \alpha}\right)-\frac{\partial \eta}{\partial \theta}(\bar{F}, \bar{\theta})(\theta-\bar{\theta}) \\
\Sigma_{j \beta}(F, \theta \mid \bar{F}, \bar{\theta}) & :=\Sigma_{j \beta}(F, \theta)-\Sigma_{j \beta}(\bar{F}, \bar{\theta})-\frac{\partial \Sigma_{i \alpha}}{\partial F_{j \beta}}(\bar{F}, \bar{\theta})\left(F_{i \alpha}-\bar{F}_{i \alpha}\right)-\frac{\partial \Sigma_{j \beta}}{\partial \theta}(\bar{F}, \bar{\theta})(\theta-\bar{\theta}) .
\end{aligned}
$$

Let us just comment that in (29), the effect of viscous dissipation and heat conduction is captured respectively by the terms

$$
\begin{aligned}
D_{v} & :=\theta \bar{\theta}\left(\frac{\nabla v}{\theta}-\frac{\nabla \bar{v}}{\bar{\theta}}\right):\left(\frac{Z}{\theta}-\frac{\bar{Z}}{\bar{\theta}}\right) \\
D_{q} & :=\left(\bar{\theta} \frac{Q}{\theta}-\theta \frac{\bar{Q}}{\bar{\theta}}\right) \cdot\left(\frac{\nabla \theta}{\theta}-\frac{\nabla \bar{\theta}}{\bar{\theta}}\right) .
\end{aligned}
$$

Moreover it can be easily checked that the same relative entropy formula can be derived for the case that we compare two different constitutive theories that have the same thermoelastic part but different viscoelastic and heat conduction formulas.

Acknowledgements Christoforou would like to thank the organizers of XVI International Conference on Hyperbolic Problems Theory, Numerics, Applications(Hyp2016) that took place in Aachen from August 1st until 5th of 2016 for the invitation and the warm hospitality.

\section{References}

1. J.J. Alibert and G. Bouchitté: Non-uniform integrability and generalized Yound measures, $J$. Convex Analysis 4 (1997), 129-147.

2. J.M. Ball: A version of the fundamental theorem for Young measures, In PDEs and Continuum Models of Phase Transitions, M. RASClE, D. SerRe, M. Slemrod, eds., Lecture Notes in Physics, Vol. 344, Springer, New York, 1988, pp. 207-215.

3. Y. Brenier, C. De Lellis and L. Szèkelyhidi Jr.,: Weak-strong uniqueness for measure-valued solutions, Comm. Math. Physics 305 (2011), 351-361.

4. K. Choi and A Vasseur: Short-time stability of scalar viscous shocks in the inviscid limit by the relative entropy method. SIAM J. Math. Anal. 47 (2015), 1405-1418.

5. C. Christoforou and A. Tzavaras: Relative entropy for hyperbolic-parabolic systems and application to the constitutive theory of thermoviscoelasticity preprint 2016. 
6. B.D. Coleman and W. Noll: The thermodynamics of elastic materials with heat conduction and viscosity. Arch. Rational Mech. Anal. 13 (1963), $167-178$.

7. B.D. Coleman and V.J. Mizel: Existence of caloric equations of state in thermodynamics. $J$. Chem. Physics 40 (1964), 1116 - 1125.

8. C. M. Dafermos: The second law of thermodynamics and stability. Arch. Rational Mech. Anal. 70 (1979), 167-179.

9. C. M. Dafermos: Stability of motions of thermoelastic fluids. J. Thermal Stresses 2 (1979), $127-134$.

10. C.M. Dafermos: Hyperbolic Conservation Laws in Continuum Physics, Third Edition. Grundlehren der Mathematischen Wissenschaften, 325. Springer Verlag, Berlin, 2010.

11. S. Demoulini, D.M.A. Stuart, A.E. Tzavaras: Weak-strong uniqueness of dissipative measurevalued solutions for polyconvex elastodynamics, Arch. Rational Mech. Analysis 205 (2012), 927-961.

12. R. J. DiPerna: Uniqueness of solutions to hyperbolic conservation laws, Indiana Univ. Mah. J. 28 (1979), 137-187.

13. R. J. DiPerna and A. J. Majda: Oscillations and concentrations in weak solutions of the incompressible Euler equations, Commun. Math. Phys. 108 (1987), 667-689.

14. E. Feireisl and A. Novotny: Weak-strong uniqueness property for the full Navier-StokesFourier system, Arch. Rational Mech. Anal. 204 (2012), 683-706.

15. E. Feireisl: Asymptotic analysis of compressible, viscous and heat conducting fluids. Nonlinear dynamics in partial differential equations, pp. 133, Adv. Stud. Pure Math., 64, Math. Soc. Japan, Tokyo, 2015.

16. U. S. Fjordholm, R. Käppeli, S. Mishra and E. Tadmor: Construction of approximate entropy measure-valued solutions for hyperbolic systems of conservation laws. Preprint (2014).

17. K. O. Friedrichs and P. D. Lax: Systems of conservation equations with a convex extension, Proc. Nat. Acad. Sci. USA 68 (1971), 1686-1688.

18. D. Iesan: On the stability of motions of thermoelastic fluids, J. Thermal Stresses 17 (1994), pp. 409-418.

19. S. Kawashima: Systems of a hyperbolic-parabolic composite type, with applications to the equations of magnetohydrodynamics, Doctoral thesis, Kyoto University, 1984.

20. S. Kawashima: Large-time behaviour of solutions to hyperbolic-parabolic systems of conservation laws and applications, Proc. Roy. Soc. Edinburgh Sect. A 106 (1987), 169-194.

21. C. Lattanzio and A.E. Tzavaras: Structural properties of stress relaxation and convergence from viscoelasticity to polyconvex elastodynamics. Arch. Rational Mech. Anal. 180 (2006), 449-492.

22. C. Lattanzio and A.E. Tzavaras: Relative entropy in diffusive relaxation SIAM J. Math. Anal. 45 (2013), 1563-1584.

23. A. Miroshnikov and K.Trivisa: Relative entropy in hyperbolic relaxation for balance laws. Commun. Math. Sci. 12 (2014), 1017-1043.

24. D. Serre and A. Vasseur: L2-type contraction for systems of conservation laws. J. École Polytech. Math. 1 (2014), 1-28.

25. L. Tartar: Compensated compactness and applications to partial differential equations. In Nonlinear Analysis and Mechanics, R.J. Knops, ed., Heriot-Watt Symposium, Vol. IV, Pitman Research Notes in Mathematics, Pitman, Boston, 1979, pp.136-192.

26. C. Truesdell and W. Noll: The non-linear field theories of mechanics. Handbuch der Physik, Bd. III/3, pp. 1-602, Springer Verlag, Berlin 1965.

27. A. E. Tzavaras: Relative entropy in hyperbolic relaxation. Commun. Math. Sci. 3 (2005), 119-132.

28. Tai-Ping Liu and Yanni Zeng: Large time behavior of solutions for general quasilinear hyperbolic-parabolic systems of conservation laws. Mem. Amer. Math. Soc. 125 (1997), pp $1-120$. 